\title{
FINITE GROUPS ACTING ON SPHERES
}

\author{
JOHN DAVID MILLER
}

\begin{abstract}
This paper classifies smooth actions of a finite group $G$ acting on a sphere of sufficiently high dimension with precisely two fixed points (and the action free elsewhere) by relating the group action to an element of the group's Whitehead group. Essentially, if that element is zero, the action corresponds to a free action on a sphere of dimension one less. If not, what we call the "double" of the action is. An example is constructed to show that the various cases can occur.
\end{abstract}

1. Introduction. Let $S^{n}$ be a smooth sphere of dimension $n, n>5$. Let $G$ be a finite group acting smoothly on $S^{n}$ with precisely two fixed points and the action free elsewhere. For certain groups, our aim is to classify all such actions by relating them to the torsion invariant of Whitehead for $h$-cobordisms in the following manner: First, remove an invariant cellular neighborhood of each fixed point and consider the orbit space defined by the action of $G$. The orbit space is seen to be an $h$-cobordism, and if this $h$-cobordism is a product, the action of $G$ on $S^{n}$ is equivariantly equivalent to a suspension of a free action on a sphere of dimension $n-1$. This is our theorem. If it is not, then what we call the "double" of $G$ is. This is our proposition. We also construct an example to show that these cases occur.

2. Preliminaries. In this section we collect some elementary facts and definitions for future reference.

Let $G$ be a finite group with the discrete topology. A differentiable action of $G$ on a smooth manifold is a mapping $\eta: G \times M \rightarrow M$ such that

(i) $\eta(e, x)=x$ for $e$ the identity;

(ii) $\eta\left(g_{1}, \eta\left(g_{2}, x\right)\right)=\eta\left(g_{1} g_{2}, x\right)$;

(iii) $\eta$ is a differentiable map.

$\eta(g, x)$ will be written as $g \cdot x$. A set $S$ is invariant under the action of $G$ if $g \cdot s \in S$ for each $g \in G$ and $s \in S$.

For a fixed $x \in M$, the isotropy subgroup of $x$, denoted by $G_{x}$, is that

Received by the editors January 4, 1971 and, in revised form, May 4, 1971.

AMS 1970 subject classifications. Primary 57E25, 57D80; Secondary 55C20, 55C35.

Key words and phrases. Group acting smoothly on $S^{n}, h$-cobordism, Whitehead torsion, Stalling's Theorem.

(c) American Mathematical Society 1972 
subgroup of $G$ defined by $G_{x}=\{g: g \cdot x=x\}$. If $G_{x}=\{e\}$ then $G$ is said to act freely at $x$. A point $x \in M$ is called a fixed point under the action of $G$ if $G_{x}=G$.

Next we define an equivalence relation $\sim$ on $M$ by $x \sim y$ if there exists a $g \in G$ such that $y=g \cdot x$. The equivalence class of $x$ is called the orbit of $x$ and the space defined by the equivalence relation the orbit space. The orbit space will be denoted by $M / G$.

Let $G^{\prime}$ be another finite group and let $G^{\prime}$ act differentiably on a manifold $M^{\prime}$. Suppose $\lambda: G \rightarrow G^{\prime}$ is a homomorphism. A $\lambda$-equivariant map $f: M \rightarrow M^{\prime}$ is a continuous map such that $f(g \cdot x)=\lambda(g) \cdot f(x)$. It is clear that $f$ induces a continuous mapping $\bar{f}: M / G \rightarrow M^{\prime} / G^{\prime}$ so that the following diagram

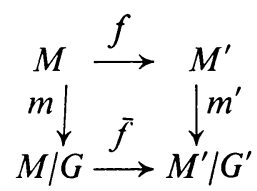

is commutative. ( $m$ and $m^{\prime}$ are the natural mappings.) If $\lambda$ is an isomorphism and $f$ is a diffeomorphism, then the actions are equivariantly equivalent and $f$ is called a $\lambda$-equivariant equivalence.

Now suppose $M$ and $M^{\prime}$ are simply connected and $G$ acts freely on $M$ and $M^{\prime}$. Suppose further there exists a diffeomorphism $\bar{f}: M / G \rightarrow M^{\prime} / G$. Then if $G$ is finite there exists an automorphism $\lambda: G \rightarrow G$ and a $\lambda$-equivariant equivalence $f$ between $M$ and $M^{\prime}$. This follows from the fiber map theorem (see e.g., p. 90 of [2]) and since an injective homomorphism between the same finite group is an automorphism.

Finally, let $G$ operate orthogonally on an $n$-sphere $S$. We define an action of $G$ on $S \times I$, where $I$ is the unit interval, by $g \cdot(x, t)=(g \cdot x, t)$. Since $S$ is the boundary of an $(n+1)$-disk we can attach $(n+1)$-disks $D_{0}$ and $D_{1}$ to $S \times I$ along $S \times 0$ and $S \times 1$ by the appropriate inclusion mappings and then give the union, $S \times I \cup D_{0} \cup D_{1}$, a compatible smoothness structure. Clearly, this union is an $(n+1)$-sphere $S^{n+1}$. Moreover, since $G$ operates orthogonally on $S$, we can extend the action of $G$ to the attached disks by requiring $G$ to act orthogonally on them. This action of $G$ on $S^{n+1}$ is called the suspension of $G$. If $G$ acts orthogonally on another $n$ sphere $S^{\prime}$ and if $\Phi: S^{\prime} \rightarrow S$ is an equivariant diffeomorphism, then it is clear that $\Phi$ can be extended to an equivariant diffeomorphism between the suspended actions of $G$ and the resulting $(n+1)$-spheres. In $\S 3$ it will be convenient to consider the following suspension of $G$. Let $G$ act orthogonally on $n$-spheres $S$ and $S^{\prime}$ and suppose $\Phi: S \rightarrow S^{\prime}$ is an equivariant diffeomorphism. Define an action of $G$ on $S \times I_{0}$ and $S^{\prime} \times I_{1}$, where $I_{0}=$ $\left[0, \frac{1}{2}\right]$ and $I_{1}=\left[\frac{1}{2}, 1\right]$, as above. We may use $\Phi$ to glue $S \times I_{0}$ to $S^{\prime} \times I_{1}$ along $S \times \frac{1}{2}$ and $S^{\prime} \times \frac{1}{2}$ and give the union $S \times I_{0} \cup S^{\prime} \times I_{1}$ a compatible 
smoothness structure. We define an equivariant diffeomorphism $F: S \times I \rightarrow S \times I_{0} \cup S^{\prime} \times I_{1}$ by the rule:

$$
\begin{aligned}
F(x, t) & =(x, t) & & \text { if } 0 \leqq t \leqq \frac{1}{2}, \\
& =(\Phi(x), t) & & \text { if } \frac{1}{2} \leqq t \leqq 1 .
\end{aligned}
$$

Clearly $F$ can be extended to an equivariant diffeomorphism between the indicated actions of $G$ and the resulting $(n+1)$-spheres.

3. THEOREM. Let $G$ act on $S^{n}, n>5$, as in the manner of the introduction, and let the Whitehead group $\mathrm{Wh}(G)$ be such that

(i) every element of $\mathrm{Wh}(G)$ is self-conjugate,

(ii) $\mathrm{Wh}(G)$ does not have elements of order 2.

Then the action of $G$ on $S^{n}$ is equivariantly equivalent to a suspension of $a$ free action on a sphere of dimension $n-1$. In effect, all such actions on spheres of sufficiently high dimensions are classified in view of Zassenhaus's work in [6].

Proof. A theorem due to Bochner asserts that there is an invariant cellular neighborhood of a fixed point upon which the action of $G$ reduces to a linear action (see e.g., p. 206 of [5]); thus, by the usual averaging process, we may assume that the given action of $G$ induces an orthogonal action on some invariant cellular neighborhood of a fixed point.

Now remove the interiors of such neighborhoods of the two fixed points and there results a manifold $W$ with boundary the disjoint union $S_{1} \cup S_{2}$ of two spheres. Evidently $W$ is diffeomorphic to $S_{1} \times I$.

Next, since the given action of $G$ on $S^{n}$ induces an orthogonal action on $S_{1}$, we define an action of $G$ on $S_{1} \times I$ by the rule:

$$
g \cdot(x, t)=(g \cdot x, t) .
$$

Observe that $G$ acts freely on $W$ without switching its boundary components as it does on $S_{1} \times I$. Hence the triples $\left(W / G ; S_{1} / G, S_{2} / G\right)$ and $\left(S_{1} / G \times I ; S_{1} / G, S_{1} / G\right)$ are manifold triads, and they are also $h$-cobordisms since the appropriate inclusion mappings clearly induce isomorphisms of homotopy groups.

We now show that under the conditions of the hypothesis $W / G$ is diffeomorphic to $S_{1} / G \times I$. If $n$ is odd, then the dimension of $S_{1}$ is even. But $G$ acts freely on $S_{1}$ and so, according to Proposition 18.3 on p. 291 of [2], $G$ must be a cyclic group of order 2 . In this case, $\mathrm{Wh}(G)=0$ and it follows that $W / G$ is diffeomorphic to $S_{1} / G \times I$. If $n$ is even, the Whitehead torsion $\tau\left(W / G, S_{1} / G\right)$ satisfies $2 \tau\left(W / G, S_{1} / G\right)=0$ by the duality theorem of [4] and the hypothesis on the self-conjugacy of the elements of $\mathrm{Wh}(G)$. The hypothesis that $\mathrm{Wh}(G)$ does not have the elements of order 2 implies 
that $\tau\left(W / G, S_{1} / G\right)=0$. This in turn implies that $W / G$ is diffeomorphic to $S_{1} / G \times I$. Hence, in either case, from $\S 2$, there are a diffeomorphism $H: W \rightarrow S_{1} \times I$ and an automorphism $\lambda: G \rightarrow G$ such that $H(g \cdot x)=\lambda(g) \cdot H(x)$. Moreover, since $H_{\mid S_{1}}: S_{1} \rightarrow S_{1} \times 0$ can be assumed to be the identity that forces $\lambda$ to be the identity automorphism.

Finally, we replace the cellular neighborhoods of the fixed points, and we attach two $n$-disks $D_{1}$ and $D_{2}$ to $S_{1} \times I$ along its boundary and extend the action of $G$ on the resulting $n$-sphere as before. That $H$ can be extended to an equivariant diffeomorphism at the "end" containing $S_{1} \times 0$ follows from the fact that $H_{\mid S_{1}}$ is the identity; that it can be extended from the other end follows from the Atiyah-Bott Theorem (see p. 409 of [4]), which implies that the induced orthogonal action on $D_{1}$ is equivalent to its induced action on $D_{2}$, and so the equivalence between the suspensions of $G$ constructed at the end of $\$ 2$ may be used. Hence the theorem follows.

REMARK 1. If $G$ is a finite abelian group, then it is known that condition (ii) placed on $\mathrm{Wh}(G)$ holds. (See Lemma 6.7 of [4].)

REMARK 2. If $G$ is any finite group acting on $S^{n}$ in the manner of the introduction with an element of order 2 , then it has precisely one since $G$ must act freely on the boundary of an invariant cellular neighborhood of a fixed point. (See e.g. the remark on p. 624 of [3].)

REMARK 3. If $G$ is a finite abelian group, then according to Theorem 10.8 of [1] if $G$ does not have elements of order 2 neither does $\mathrm{Wh}(G)$.

REMARK 4. The hypothesis that $G$ has precisely two fixed points and acts freely elsewhere may be dropped to read: " $G$ has finitely many fixed points and acts freely elsewhere", for it is well known that a periodic transformation of prime period on a homology $n$-sphere has as fixed point set a homology $r$-sphere.

We close out this section by showing the necessity of $\mathrm{Wh}(G)$ lacking elements of order 2 .

Let $G$ be abelian and let it act orthogonally and fixed point free on $S^{n-1}$. Consider the manifold $M=S^{n-1} / G$. By Stalling's Theorem [4], for any $\tau \in \mathrm{Wh}(G)$, there is an $h$-cobordism $W$ with $\tau=\tau(W, M)$. Let $W^{\prime}$ be the covering manifold of $W$, which is an $h$-cobordism with $(n-1)$-spheres as boundary. Now $G$ acts freely on $W^{\prime}$ carrying components of the boundary into the same components. Since $W^{\prime}$ is diffeomorphic to $S^{n-1} \times I$ and the action of $G$ is orthogonal on $S^{n-1}$, we can "cap" the boundary by $n$-disks, thus obtaining an $n$-sphere $S^{n}$, and extend the action of $G$ onto $S^{n}$. Clearly this action of $G$ on $S^{n}$ is of the sort considered in $\S 1$, but it is not equivalent to a suspended action if $\tau$ is an element of order 2 .

4. Proposition. There is still the nagging desire to treat the case when $\tau\left(W / G, S_{1} / G\right)$ is an element of order 2 . Naturally in this case the action 
of $G$ on $S^{n}$ cannot be equivariantly equivalent to a suspension of a free action on $S_{1}$. We note, however, that $n$ in this case must be even, since for $n$ odd $G$ must be $Z_{2}$ which implies $\mathrm{Wh}(G)=0$. Thus the situation is this: $W$ is an even dimensional manifold whose boundary components are two spheres $S_{1}$ and $S_{2}$ and the action of $G$ on $W$ carries $S_{1}$ into $S_{1}$. Moreover, the elements of $\mathrm{Wh}(G)$ are self-conjugate.

Now let $2 W$ denote the "double" of $W$, which is formed by pasting together two copies of $W$ along one boundary component. The action of $G$ on $W$ induces a natural action on $2 W$ which we shall call the "double" of $G$. This action is not, as we shall see, equivariantly equivalent to the given action of $G$ on $W .2 W$, of course, is diffeomorphic to $S_{1} \times I$.

Finally, let us recall that the Whitehead torsion $\tau\left(2 W / G, S_{1} / G\right)$ is given by

$$
\tau\left(2 W / G, S_{1} / G\right)=\tau\left(W / G, S_{1} / G\right)+(-1)^{n-1} \bar{\tau}\left(W / G, S_{1} / G\right)
$$

(see $\S 11$ of [4]). Since $n$ is even and the elements of $\mathrm{Wh}(G)$ are selfconjugate, this implies that $\tau\left(2 W / G, S_{1} / G\right)=0$; hence, $2 W / G$ is diffeomorphic to $S_{1} / G \times I$. Thus, using our previous results and the above discussion, we have shown the

Proposition. Let $G$ be a finite group acting smoothly on $S^{n}, n \geqq 6$. Suppose further that the action has finitely many fixed points with the action free elsewhere. Then, if the elements of $\mathrm{Wh}(G)$ are self-conjugate, either the action is equivariantly equivalent to a suspension of a free action on a sphere of dimension $n-1$ or else the "double" of $G$ is. The latter case can occur only when $n$ is even and $\mathrm{Wh}(G)$ has elements of order 2.

\section{REFERENCES}

1. H. Bass and M. Murthy, Grothendieck groups and Picard groups of abelian group rings, Ann. of Math. (2) 86 (1967), 16-73. MR 36 \#2671.

2. S. T. Hu, Homotopy theory, Pure and Appl. Math., vol. 8, Academic Press, New York, 1959. MR 21 \#5186.

3. J. W. Milnor, Groups which act on $S^{n}$ without fixed points, Amer. J. Math. 79 (1957), 623-630. MR 19, 761.

4. - Whitehead torsion, Bull. Amer. Math. Soc. 72 (1966), 358-426. MR 33 \#4922.

5. D. Montgomery and L. Zippin, Topological transformation groups, Interscience, New York, 1955. MR 17, 383.

6. H. Zassenhaus, Über endliche Fastkorper, Abh. Math. Sem. Univ. Hamburg 11 (1936), 187-220.

Department of Mathematics, Texas Tech University, Lubbock, Texas 79406 